ERRATUM

\title{
Evaluation of indeterminate biliary strictures
}

Christopher L. Bowlus, Kristin A. Olson \& M. Eric Gershwin

Nature Reviews Gastroenterology \& Hepatology 13, 28-37 (2016)

In the version of this article originally published online and in print, the main text incorrectly specified endoscopic ultrasonography (EUS)-guided fine-needle aspiration (FNA) when referring to reference 104 and the potential for seeding of tumours, when the study discussed FNA in general. The sentences should have read: "Despite the potential benefits of EUS-guided FNA, concerns have been raised about FNA in general and potential seeding along the needle tract resulting in peritoneal metastases ${ }^{103}$. In a study of 16 patients who had an FNA before pre-liver-transplantation laparoscopic staging, five of six patients with a FNA demonstrating malignancy upon cytological examination had peritoneal metastases

identified ${ }^{104}$." This error has been corrected in the HTML and PDF versions of the article. 